Article

\title{
A Mixed Element Algorithm Based on the Modified L1 Crank-Nicolson Scheme for a Nonlinear Fourth-Order Fractional Diffusion-Wave Model
}

\author{
Jinfeng Wang ${ }^{1}$, Baoli Yin ${ }^{2}$, Yang Liu ${ }^{2, *} \mathbb{( D}$, Hong $\mathrm{Li}^{2, *} \mathbb{C}$ and Zhichao Fang ${ }^{2}(\mathbb{D}$ \\ 1 School of Statistics and Mathematics, Inner Mongolia University of Finance and Economics, \\ Hohhot 010070, China; w45j85f@163.com \\ 2 School of Mathematical Sciences, Inner Mongolia University, Hohhot 010021, China; \\ baolimath@126.com (B.Y.); zcfang@imu.edu.cn (Z.F.) \\ * Correspondence: mathliuyang@imu.edu.cn (Y.L.); smslh@imu.edu.cn (H.L.)
}

check for updates

Citation: Wang, J.; Yin, B.; Liu, Y.; Li, H.; Fang, Z. A Mixed Element Algorithm Based on the Modified L1 Crank-Nicolson Scheme for a Nonlinear Fourth-Order Fractional Diffusion-Wave Model. Fractal Fract. 2021, 5, 274. https://doi.org/ 10.3390 / fractalfract 5040274

Academic Editors: Carlo Cattani and Haci Mehmet Baskonus

Received: 15 October 2021

Accepted: 10 December 2021

Published: 14 December 2021

Publisher's Note: MDPI stays neutral with regard to jurisdictional claims in published maps and institutional affiliations.

Copyright: (c) 2021 by the authors. Licensee MDPI, Basel, Switzerland. This article is an open access article distributed under the terms and conditions of the Creative Commons Attribution (CC BY) license (https:/ / creativecommons.org/licenses/by/ $4.0 /)$.

\begin{abstract}
In this article, a new mixed finite element (MFE) algorithm is presented and developed to find the numerical solution of a two-dimensional nonlinear fourth-order Riemann-Liouville fractional diffusion-wave equation. By introducing two auxiliary variables and using a particular technique, a new coupled system with three equations is constructed. Compared to the previous space-time high-order model, the derived system is a lower coupled equation with lower time derivatives and second-order space derivatives, which can be approximated by using many time discrete schemes. Here, the second-order Crank-Nicolson scheme with the modified L1-formula is used to approximate the time direction, while the space direction is approximated by the new MFE method. Analyses of the stability and optimal $L^{2}$ error estimates are performed and the feasibility is validated by the calculated data.
\end{abstract}

Keywords: fourth-order fractional diffusion-wave equation; modified L1-formula; mixed element method; a priori error estimates

\section{Introduction}

Fourth-order fractional partial differential equations (PDEs) including fourth-order fractional subdiffusion models [1-3] and fourth-order fractional diffusion-wave models $[2,4,5]$ can be founded in many fields of science and engineering. Thus far, there have been many efficient numerical algorithms for solving linear or nonlinear fourth-order fractional subdiffusion and diffusion-wave models. Liu et al. [6], Liu et al. [7], and Liu et al. [8] considered different mixed element methods to solve fourth-order nonlinear fractional subdiffusion models with the first-order time derivative and developed numerical theories including stability and convergence. Liu et al. [3] introduced a mixed element algorithm with a new approximation of the fractional derivative. Ji et al. [9], Ran et al. [10], Nandal and Pandey [11], Sun et al. [12], and Huang et al. [13] considered some difference schemes for linear or nonlinear fourth-order fractional diffusion or diffusion-wave models. Abbaszadeh and Dehghan [14] studied the direct meshless local Petrov-Galerkin method for solving fourth-order reaction-diffusion problems with a time-fractional derivative. Yang et al. [15] and Zhang et al. [16] found the numerical solutions for a fourth-order fractional model by using the orthogonal spline collocation method. Tariq and Akram [17] considered a quintic spline technique to solve a fourth-order time-fractional subdiffusion model. Guo et al. [18] and Du et al. [19] studied the LDG methods for solving some timefractional subdiffusion models with fourth-order spatial derivative terms, respectively. In [1], Nikan et al. developed a local radial basis function generated by the finite difference scheme for a time-fractional fourth-order reaction-diffusion model. In [5], Jafari et al. solved a fourth-order fractional diffusion-wave equation by the decomposition method. Hu 
and Zhang [2] implemented numerical calculations via finite difference methods for fourthorder time fractional subdiffusion and diffusion-wave models. Li and Wong [20] developed an efficient numerical algorithm for a fourth-order time-fractional diffusion-wave model.

Here, we propose a new mixed element algorithm to solve the following nonlinear fourth-order time-fractional diffusion-wave model:

$$
\left\{\begin{array}{l}
\frac{\partial^{2} u}{\partial t^{2}}+\frac{\partial_{R L}^{\beta} u}{\partial t^{\beta}}+\frac{\partial u}{\partial t}+\triangle^{2} u-\triangle f(u)=g(\mathbf{x}, t),(\mathbf{x}, t) \in \Omega \times J \\
u(\mathbf{x}, t)=\Delta u(\mathbf{x}, t)=0, t \in \bar{J}, \\
u(\mathbf{x}, 0)=0, \frac{\partial u}{\partial t}(\mathbf{x}, 0)=u_{1}(\mathbf{x}), \mathbf{x} \in \bar{\Omega}
\end{array}\right.
$$

where $\Omega \subset R^{d}(d \leq 2)$ and $J=(0, T]$ with $0<T<\infty$ are the spatial domain and time interval, respectively. $u_{1}(\mathbf{x})$ is an initial value function, $g(\mathbf{x}, t)$ is a given source term, $f(u)$ is a polynomial function or bounded function on $u$ satisfying $f \in C^{2}(R)$, and the Riemann-Liouville fractional derivative is defined by

$$
\frac{\partial_{R L}^{\beta} u}{\partial t^{\beta}}=\frac{1}{\Gamma(2-\beta)} \frac{\partial^{2}}{\partial t^{2}} \int_{0}^{t} \frac{u(s) d s}{(t-s)^{\beta-1}}, 1<\beta<2,
$$

where the nonlinear fourth-order fractional diffusion-wave model (1) can be generated by the classical fourth-order hyperbolic wave equation. When $\beta \rightarrow 1$ or 2 and $f(u)=u^{3}-u$, the model (1) can be reduced to an important Cahn-Hilliard equation model [21].

Recently, Zeng and Li [22] developed a new Crank-Nicolson scheme based on a modified L1-formula, whose coefficients are different from the famous L1-formula (see [23,24] for the fractional parameter $\alpha \in(0,1)$ ). One should note that this modified L1-formula can only approximate the Caputo or Riemann-Liouville fractional derivative with parameter $\alpha \in(0,1)$, and it cannot approximate the case $\beta \in(1,2)$. Here, we will develop the modified L1-formula for the case of $\beta \in(1,2)$ by using some techniques.

In this article, by introducing two auxiliary functions and using some techniques, we propose a new mixed element algorithm. Here, our major contributions are as follows: (1) by the introduction of two auxiliary functions, we reduce the nonlinear fourth-order time-fractional diffusion-wave model to a low-order coupled system; (2) we turn order $\beta \in(1,2)$ into order $\alpha \in(0,1)$ for the Riemann-Liouville fractional derivative; (3) we approximate the derived coupled system with a fractional derivative with order $\alpha \in(0,1)$ by the modified $L 1$ Crank-Nicolson scheme with the developed new mixed element method; (4) we derive the stability of the new mixed element scheme and optimal error estimates in the $L^{2}$-norm for three functions.

The structure of this article is as follows: in Section 2, we provide some numerical approximation formulas, propose a new mixed element scheme, and prove the stability of the derived scheme; in Section 3, we derive optimal error estimates for three variables; in Section 4, some numerical data are computed and discussed; Finally, in Section 5, we give some concluding remarks.

\section{Numerical Approximation and Stability}

Based on the relation between the Riemann-Liouville fractional derivative and Caputo fractional derivative, we take $\alpha=\beta-1$ and $v=\frac{\partial u}{\partial t}$ to obtain

$$
\begin{aligned}
\frac{\partial_{R L}^{\beta} u}{\partial t^{\beta}} & =\frac{1}{\Gamma(2-\beta)} \int_{0}^{t} \frac{\frac{\partial^{2} u(s)}{\partial s^{2}} d s}{(t-s)^{\beta-1}}+\frac{u(0)}{\Gamma(1-\beta)} t^{-\beta}+\frac{\frac{\partial u(0)}{\partial t}}{\Gamma(2-\beta)} t^{1-\beta} \\
& =\frac{1}{\Gamma(1-\alpha)} \int_{0}^{t} \frac{\frac{\partial v}{\partial s} d s}{(t-s)^{\alpha}}+\frac{v(0)}{\Gamma(1-\alpha)} t^{-\alpha} \\
& =\frac{\partial_{R L}^{\alpha} v}{\partial t^{\alpha}}, 0<\alpha<1 .
\end{aligned}
$$


Let $\sigma=\Delta u-f(u) ;(1)$ can be rewritten as the following coupled system:

$$
\left\{\begin{array}{l}
v=\frac{\partial u}{\partial t},(\mathbf{x}, t) \in \Omega \times J, \\
\frac{\partial \sigma}{\partial t}=\Delta v-f_{u}(u) v,(\mathbf{x}, t) \in \Omega \times J, \\
\frac{\partial v}{\partial t}+\frac{\partial_{R L}^{\alpha} v}{\partial t^{\alpha}}+v+\Delta \sigma=g(\mathbf{x}, t),(\mathbf{x}, t) \in \Omega \times J .
\end{array}\right.
$$

For formulating the fully discrete scheme, we insert the nodes $t_{n}=n \Delta t(n=0,1,2, \cdots, N)$ in time interval $[0, T]$, where $t_{n}$ satisfy $0=t_{0}<t_{1}<t_{2}<\cdots<t_{N}=T$ and the time step length size $\Delta t=T / N$, for some positive integer $N$. For a smooth function $\phi$ defined on the time interval $[0, T]$, we denote $\phi^{n}=\phi\left(t_{n}\right)$.

Now, we need to introduce some lemmas on integer and fractional derivatives.

Lemma 1. At $t_{k+\frac{1}{2}}$, the following relation holds:

$$
\begin{aligned}
\frac{\partial \phi}{\partial t}\left(t_{k+\frac{1}{2}}\right) & =\frac{\phi^{k+1}-\phi^{k}}{\Delta t}+O\left(\Delta t^{2}\right) \\
& \triangleq P_{\Delta t} \phi^{k+\frac{1}{2}}+O\left(\Delta t^{2}\right) .
\end{aligned}
$$

Lemma 2. At $t_{k+\frac{1}{2}}$, we have

$$
\begin{aligned}
\phi\left(t_{k+\frac{1}{2}}\right) & =\frac{\phi^{k+1}+\phi^{k}}{2}+O\left(\Delta t^{2}\right) \\
& \triangleq \phi^{k+\frac{1}{2}}+O\left(\Delta t^{2}\right)
\end{aligned}
$$

Lemma 3 ([22]). At $t_{k+\frac{1}{2}}$, the Caputo fractional derivative has the following form:

$$
\begin{aligned}
\frac{\partial_{C}^{\alpha} \phi}{\partial t^{\alpha}}\left(t_{k+\frac{1}{2}}\right)= & \frac{1}{\Gamma(1-\alpha)} \int_{0}^{t_{k+\frac{1}{2}}} \frac{\frac{\partial \phi}{\partial s} d s}{\left(t_{k+\frac{1}{2}}-s\right)^{\alpha}} \\
= & \frac{\Delta t^{-\alpha}}{\Gamma(2-\alpha)}\left[a_{0} \phi\left(t_{k+\frac{1}{2}}\right)-\sum_{j=1}^{k}\left(a_{k-j}-a_{k-j+1}\right) \phi\left(t_{j-\frac{1}{2}}\right)\right. \\
& \left.-\left(a_{k}-b_{k}\right) \phi\left(t_{\frac{1}{2}}\right)-b_{k} \phi\left(t_{0}\right)\right]+O\left(\Delta t^{2-\alpha}\right)
\end{aligned}
$$

for $k \geq 0$, we have

$$
b_{k}=2\left[\left(k+\frac{1}{2}\right)^{1-\alpha}-k^{1-\alpha}\right], a_{k-j}=\left[(k-j+1)^{1-\alpha}-(k-j)^{1-\alpha}\right] .
$$

By Lemma 3, we have

Lemma 4 ([22]). At $t_{k+\frac{1}{2}}$, the Riemann-Liouville fractional derivative has the following approximation:

$$
\begin{aligned}
\frac{\partial_{R L}^{\alpha} \phi}{\partial t^{\alpha}}\left(t_{k+\frac{1}{2}}\right)= & \frac{\Delta t^{-\alpha}}{\Gamma(2-\alpha)}\left[a_{0} \phi\left(t_{k+\frac{1}{2}}\right)-\sum_{j=1}^{k}\left(a_{k-j}-a_{k-j+1}\right) \phi\left(t_{j-\frac{1}{2}}\right)\right. \\
& \left.-\left(a_{k}-b_{k}\right) \phi\left(t_{\frac{1}{2}}\right)-\widehat{b}_{k} \phi\left(t_{0}\right)\right]+O\left(\Delta t^{2-\alpha}\right),
\end{aligned}
$$

where $\widehat{b}_{k}=b_{k}-(1-\alpha)\left(k+\frac{1}{2}\right)^{-\alpha}$. 
Remark 1. In [22], the authors provided the L1-formula above, which is different from the usual L1-formula and called the modified L1-formula.

By the approximation scheme above, we arrive at

$$
\left\{\begin{array}{l}
\text { (a) } P_{\Delta t} u^{n+\frac{1}{2}}=v^{n+\frac{1}{2}}+R_{1}^{n+\frac{1}{2}}, \\
\text { (b) } P_{\Delta t} \sigma^{n+\frac{1}{2}}=\Delta v^{n+\frac{1}{2}}-\frac{f_{u}\left(u^{n+1}\right) v^{n+1}+f_{u}\left(u^{n}\right) v^{n}}{2}+R_{2}^{n+\frac{1}{2}}, \\
\text { (c) } P_{\Delta t} v^{n+\frac{1}{2}}+\frac{\Delta t^{-\alpha}}{\Gamma(2-\alpha)}\left[a_{0} v^{n+\frac{1}{2}}-\sum_{j=1}^{n}\left(a_{n-j}-a_{n-j+1}\right) v^{j-\frac{1}{2}}\right. \\
\left.\quad-\left(a_{n}-b_{n}\right) v^{\frac{1}{2}}-\widehat{b}_{n} v^{0}\right]+v^{n+\frac{1}{2}}+\triangle \sigma^{n+\frac{1}{2}}=g\left(\mathbf{x}, t_{n+\frac{1}{2}}\right)+R_{3}^{n+\frac{1}{2}},
\end{array}\right.
$$

where

$$
\begin{aligned}
& R_{1}^{n+\frac{1}{2}}=P_{\Delta t} u^{n+\frac{1}{2}}-\frac{\partial u}{\partial t}\left(t_{n+\frac{1}{2}}\right)+\left(v\left(t_{n+\frac{1}{2}}\right)-v^{n+\frac{1}{2}}\right)=O\left(\Delta t^{2}\right) \\
& R_{2}^{n+\frac{1}{2}}=P_{\Delta t} \sigma^{n+\frac{1}{2}}-\frac{\partial \sigma}{\partial t}\left(t_{n+\frac{1}{2}}\right)+\left(\Delta v\left(t_{n+\frac{1}{2}}\right)-\Delta v^{n+\frac{1}{2}}\right) \\
& +f_{u}\left(u\left(t_{n+\frac{1}{2}}\right)\right) v\left(t_{n+\frac{1}{2}}\right)-\frac{f_{u}\left(u^{n+1}\right) v^{n+1}+f_{u}\left(u^{n}\right) v^{n}}{2}=O\left(\Delta t^{2}\right), \\
& R_{3}^{n+\frac{1}{2}}=P_{\Delta t} v^{n+\frac{1}{2}}-\frac{\partial v}{\partial t}\left(t_{n+\frac{1}{2}}\right)+O\left(\Delta t^{2-\alpha}\right)+\left(v^{n+\frac{1}{2}}-v\left(t_{n+\frac{1}{2}}\right)\right) \\
& +\left(\triangle \sigma^{n+\frac{1}{2}}-\triangle \sigma\left(t_{n+\frac{1}{2}}\right)\right)=O\left(\Delta t^{2-\alpha}\right) .
\end{aligned}
$$

For $(\varphi, \psi, \chi) \in L^{2} \times H_{0}^{1} \times H_{0}^{1}$, we have the following mixed weak formulation:

$$
\left\{\begin{array}{l}
(a)\left(P_{\Delta t} u^{n+\frac{1}{2}}, \varphi\right)=\left(v^{n+\frac{1}{2}}, \varphi\right)+\left(R_{1}^{n+\frac{1}{2}}, \varphi\right), \\
\text { (b) }\left(P_{\Delta t} \sigma^{n+\frac{1}{2}}, \psi\right)+\left(\nabla v^{n+\frac{1}{2}}, \nabla \psi\right)+\left(\frac{f_{u}\left(u^{n+1}\right) v^{n+1}+f_{u}\left(u^{n}\right) v^{n}}{2}, \psi\right)=\left(R_{2}^{n+\frac{1}{2}}, \psi\right), \\
(c)\left(P_{\Delta t} v^{n+\frac{1}{2}}, \chi\right)+\left(\frac { \Delta t ^ { - \alpha } } { \Gamma ( 2 - \alpha ) } \left[a_{0} v^{n+\frac{1}{2}}-\sum_{j=1}^{n}\left(a_{n-j}-a_{n-j+1}\right) v^{j-\frac{1}{2}}\right.\right. \\
\left.\left.-\left(a_{n}-b_{n}\right) v^{\frac{1}{2}}-\widehat{b}_{n} v^{0}\right], \chi\right)+\left(v^{n+\frac{1}{2}}, \chi\right)-\left(\nabla \sigma^{n+\frac{1}{2}}, \nabla \chi\right)=\left(g\left(\mathbf{x}, t_{n+\frac{1}{2}}\right), \chi\right)+\left(R_{3}^{n+\frac{1}{2}}, \chi\right) .
\end{array}\right.
$$

For $\left(\varphi_{h}, \psi_{h}, \chi_{h}\right) \in L_{h} \times V_{h} \times V_{h} \subset L^{2} \times H_{0}^{1} \times H_{0}^{1}$, based on the mixed weak formulation above, we formulate the following new mixed element system:

$$
\left\{\begin{array}{l}
(a)\left(P_{\Delta t} u_{h}^{n+\frac{1}{2}}, \varphi_{h}\right)=\left(v_{h}^{n+\frac{1}{2}}, \varphi_{h}\right), \\
\text { (b) }\left(P_{\Delta t} \sigma_{h}^{n+\frac{1}{2}}, \psi_{h}\right)+\left(\nabla v_{h}^{n+\frac{1}{2}}, \nabla \psi_{h}\right)+\left(\frac{f_{u}\left(u_{h}^{n+1}\right) v_{h}^{n+1}+f_{u}\left(u_{h}^{n}\right) v_{h}^{n}}{2}, \psi_{h}\right)=0 \\
(c)\left(P_{\Delta t} v_{h}^{n+\frac{1}{2}}, \chi_{h}\right)+\left(\frac { \Delta t ^ { - \alpha } } { \Gamma ( 2 - \alpha ) } \left[a_{0} v_{h}^{n+\frac{1}{2}}-\sum_{j=1}^{n}\left(a_{n-j}-a_{n-j+1}\right) v_{h}^{j-\frac{1}{2}}\right.\right. \\
\left.\left.\quad-\left(a_{n}-b_{n}\right) v_{h}^{\frac{1}{2}}-\widehat{b}_{n} v_{h}^{0}\right], \chi_{h}\right)+\left(v_{h}^{n+\frac{1}{2}}, \chi_{h}\right)-\left(\nabla \sigma_{h}^{n+\frac{1}{2}}, \nabla \chi_{h}\right)=\left(g\left(\mathbf{x}, t_{n+\frac{1}{2}}\right), \chi_{h}\right) .
\end{array}\right.
$$

Lemma 5 (See [22]). For $\widehat{b}_{n-j}$, the following important inequality holds:

$$
\frac{\Delta t^{1-\alpha}}{\Gamma(2-\alpha)} \sum_{j=0}^{n-1} \widehat{b}_{n-j} \leq \frac{C T^{1-\alpha}}{\Gamma(2-\alpha)}
$$

where $C$ is a positive constant that is independent of space-time step length sizes $h$ and $\Delta t$. 
Proof. Applying the Taylor formula, we have

$$
\begin{aligned}
& \frac{\Delta t^{1-\alpha}}{\Gamma(2-\alpha)} \sum_{j=0}^{n-1} \widehat{b}_{n-j} \\
= & \frac{\Delta t^{1-\alpha}}{\Gamma(2-\alpha)} \sum_{j=0}^{n-1}\left[2\left(n-j+\frac{1}{2}\right)^{1-\alpha}-2(n-j)^{1-\alpha}-(1-\alpha)\left(n-j+\frac{1}{2}\right)^{-\alpha}\right] \\
= & \frac{2 \Delta t^{1-\alpha}}{\Gamma(2-\alpha)} \sum_{j=0}^{n-1}(n-j)^{1-\alpha}\left[\left(1+\frac{1}{2(n-j)}\right)^{1-\alpha}-1-\frac{1-\alpha}{2\left(n-j+\frac{1}{2}\right)}\left(1+\frac{1}{2(n-j)}\right)^{1-\alpha}\right] \\
= & \frac{\Delta t^{1-\alpha}}{\Gamma(2-\alpha)} \sum_{j=0}^{n-1}(n-j)^{1-\alpha}\left[\frac{1-\alpha}{2(n-j)}+\frac{(1-\alpha) \alpha}{2 !}\left(1+\kappa \frac{1}{2(n-j)}\right)^{1-\alpha} \frac{1}{4(n-j)^{2}}\right. \\
& \left.-\frac{1-\alpha}{2\left(n-j+\frac{1}{2}\right)}\left(1+\frac{1-\alpha}{2(n-j)}+\frac{(1-\alpha) \alpha}{2 !}\left(1+\kappa \frac{1}{2(n-j)}\right)^{1-\alpha} \frac{1}{4(n-j)^{2}}\right)\right] \\
= & \frac{\Delta t^{1-\alpha}}{\Gamma(2-\alpha)} \sum_{j=0}^{n-1}(n-j)^{1-\alpha}\left[\frac{1-\alpha}{2(n-j)}-\frac{1-\alpha}{2\left(n-j+\frac{1}{2}\right)}+O\left(\frac{1}{(n-j)^{2}}\right)\right] \\
= & \frac{\Delta t^{1-\alpha}}{\Gamma(2-\alpha)} \sum_{j=0}^{n-1}(n-j)^{1-\alpha}\left[\frac{1-\alpha}{2\left(n-j+\frac{1}{2}\right)(n-j)}+O\left(\frac{1}{(n-j)^{2}}\right)\right] .
\end{aligned}
$$

Noting that $\Delta t=\frac{T}{N}$ and $n-j \leq N$, we have

$$
\begin{aligned}
& \frac{\Delta t^{1-\alpha}}{\Gamma(2-\alpha)} \sum_{j=0}^{n-1}(n-j)^{1-\alpha}\left[\frac{1-\alpha}{2\left(n-j+\frac{1}{2}\right)(n-j)}+O\left(\frac{1}{(n-j)^{2}}\right)\right] \\
= & \frac{1}{\Gamma(2-\alpha)} \sum_{j=0}^{n-1} T^{1-\alpha}\left(\frac{n-j}{N}\right)^{1-\alpha}\left[\frac{1-\alpha}{2\left(n-j+\frac{1}{2}\right)(n-j)}+O\left(\frac{1}{(n-j)^{2}}\right)\right] \\
\leq & \frac{T^{1-\alpha}}{\Gamma(2-\alpha)} \sum_{j=0}^{n-1}\left[\frac{1}{(n-j)^{2}}+O\left(\frac{1}{(n-j)^{2}}\right)\right] \leq \frac{C T^{1-\alpha}}{\Gamma(2-\alpha)} \sum_{n=1}^{+\infty} \frac{1}{n^{2}} \leq \frac{C T^{1-\alpha}}{\Gamma(2-\alpha)} .
\end{aligned}
$$

Substitute (15) into (14) to obtain the conclusion.

Next, we will prove the stability.

Theorem 1. For $n \geq 0$, the stability for the fully discrete system (12) holds:

$$
\begin{aligned}
& \text { (a). }\left\|v_{h}^{n+1}\right\|+\left\|\sigma_{h}^{n+1}\right\|+\left(\frac{\Delta t^{1-\alpha}}{\Gamma(2-\alpha)} \sum_{j=1}^{n+1} a_{n-j+1}\left\|v_{h}^{j-\frac{1}{2}}\right\|^{2}\right)^{\frac{1}{2}} \\
\leq & C\left(\left\|v_{h}^{0}\right\|+\left\|\sigma_{h}^{0}\right\|+\max _{0 \leq j \leq n}\left\{\left\|g\left(x, t_{j+\frac{1}{2}}\right)\right\|\right\}\right), \\
& \text { (b). }\left\|u_{h}^{n+1}\right\| \leq C\left(\left\|u_{h}^{0}\right\|+\left\|v_{h}^{0}\right\|+\left\|\sigma_{h}^{0}\right\|+\max _{0 \leq j \leq n}\left\{\left\|g\left(x, t_{j+\frac{1}{2}}\right)\right\|\right\}\right) .
\end{aligned}
$$

Proof. In (12) (a), we take $\varphi_{h}=u_{h}^{n+\frac{1}{2}}$, and use Cauchy-Schwarz inequality as well as Young inequality to obtain

$$
\begin{aligned}
\frac{1}{2}\left(\left\|v_{h}^{n+\frac{1}{2}}\right\|^{2}+\left\|u_{h}^{n+\frac{1}{2}}\right\|^{2}\right) \geq\left(v_{h}^{n+\frac{1}{2}}, u_{h}^{n+\frac{1}{2}}\right) & =\left(P_{\Delta t} u_{h}^{n+\frac{1}{2}}, u_{h}^{n+\frac{1}{2}}\right) \\
& \geq \frac{\left\|u_{h}^{n+1}\right\|^{2}-\left\|u_{h}^{n}\right\|^{2}}{2 \Delta t} .
\end{aligned}
$$


In (12) (b), set $\psi_{h}=\sigma_{h}^{n+\frac{1}{2}}$ and make use of Cauchy-Schwarz inequality to arrive at

$$
\begin{aligned}
& \left(\nabla v_{h}^{n+\frac{1}{2}}, \nabla \sigma_{h}^{n+\frac{1}{2}}\right) \\
= & -\left(P_{\Delta t} \sigma_{h}^{n+\frac{1}{2}}, \sigma_{h}^{n+\frac{1}{2}}\right)-\left(\frac{f_{u}\left(u_{h}^{n+1}\right) v_{h}^{n+1}+f_{u}\left(u_{h}^{n}\right) v_{h}^{n}}{2}, \sigma_{h}^{n+\frac{1}{2}}\right) \\
\leq & -\frac{\left\|\sigma_{h}^{n+1}\right\|^{2}-\left\|\sigma_{h}^{n}\right\|^{2}}{2 \Delta t}+\frac{1}{2}\left(\left\|f_{u}\left(u_{h}^{n+1}\right)\right\|_{\infty}\left\|v_{h}^{n+1}\right\|+\left\|f_{u}\left(u_{h}^{n}\right)\right\|_{\infty}\left\|v_{h}^{n}\right\|\right)\left\|\sigma_{h}^{n+\frac{1}{2}}\right\| \\
\leq & -\frac{\left\|\sigma_{h}^{n+1}\right\|^{2}-\left\|\sigma_{h}^{n+1}\right\|^{2}}{2 \Delta t}+C\left(\left\|v_{h}^{n+1}\right\|^{2}+\left\|v_{h}^{n}\right\|^{2}+\left\|\sigma_{h}^{n+1}\right\|^{2}+\left\|\sigma_{h}^{n}\right\|^{2}\right) .
\end{aligned}
$$

In (12) (c), set $\chi_{h}=v_{h}^{n+\frac{1}{2}}$ and use Cauchy-Schwarz inequality to obtain

$$
\begin{aligned}
& -\left(\nabla \sigma_{h}^{n+\frac{1}{2}}, \nabla v_{h}^{n+\frac{1}{2}}\right) \\
= & -\left(P_{\Delta t} v_{h}^{n+\frac{1}{2}}, v_{h}^{n+\frac{1}{2}}\right)-\left(\frac { \Delta t ^ { - \alpha } } { \Gamma ( 2 - \alpha ) } \left[a_{0} v_{h}^{n+\frac{1}{2}}-\sum_{j=1}^{n}\left(a_{n-j}-a_{n-j+1}\right) v_{h}^{j-\frac{1}{2}}\right.\right. \\
& \left.\left.-\left(a_{n}-b_{n}\right) v_{h}^{\frac{1}{2}}-b_{n} v_{h}^{0}\right], v_{h}^{n+\frac{1}{2}}\right)-\left\|v_{h}^{n+\frac{1}{2}}\right\|^{2}+\left(g\left(\mathbf{x}, t_{n+\frac{1}{2}}\right), v_{h}^{n+\frac{1}{2}}\right) \\
\leq & -\frac{\left\|v_{h}^{n+1}\right\|^{2}-\left\|v_{h}^{n}\right\|^{2}}{2 \Delta t}-\left(\frac { \Delta t ^ { - \alpha } } { \Gamma ( 2 - \alpha ) } \left[a_{0} v_{h}^{n+\frac{1}{2}}-\sum_{j=1}^{n}\left(a_{n-j}-a_{n-j+1}\right) v_{h}^{j-\frac{1}{2}}\right.\right. \\
& \left.\left.-\left(a_{n}-b_{n}\right) v_{h}^{\frac{1}{2}}-\widehat{b}_{n} v_{h}^{0}\right], v_{h}^{n+\frac{1}{2}}\right)-\frac{1}{2}\left\|v_{h}^{n+\frac{1}{2}}\right\|^{2}+\frac{1}{2}\left\|g\left(\mathbf{x}, t_{n+\frac{1}{2}}\right)\right\|^{2} .
\end{aligned}
$$

Add (18) and (19) to obtain

$$
\begin{aligned}
& \frac{\left\|v_{h}^{n+1}\right\|^{2}-\left\|v_{h}^{n}\right\|^{2}}{2 \Delta t}+\frac{\left\|\sigma_{h}^{n+1}\right\|^{2}-\left\|\sigma_{h}^{n}\right\|^{2}}{2 \Delta t}+\frac{1}{2}\left\|v_{h}^{n+\frac{1}{2}}\right\|^{2} \\
\leq & -\left(\frac{\Delta t^{-\alpha}}{\Gamma(2-\alpha)}\left[a_{0} v_{h}^{n+\frac{1}{2}}-\sum_{j=1}^{n}\left(a_{n-j}-a_{n-j+1}\right) v_{h}^{j-\frac{1}{2}}-\left(a_{n}-b_{n}\right) v_{h}^{\frac{1}{2}}-\widehat{b}_{n} v_{h}^{0}\right], v_{h}^{n+\frac{1}{2}}\right) \\
& +C\left(\left\|v_{h}^{n+1}\right\|^{2}+\left\|v_{h}^{n}\right\|^{2}+\left\|\sigma_{h}^{n+1}\right\|^{2}+\left\|\sigma_{h}^{n}\right\|^{2}+\left\|g\left(\mathbf{x}, t_{n+\frac{1}{2}}\right)\right\|^{2}\right) .
\end{aligned}
$$

Refer to Lemma 4.2 in [22] to easily obtain

$$
\begin{aligned}
& -\left(\frac{\Delta t^{-\alpha}}{\Gamma(2-\alpha)}\left[a_{0} v_{h}^{n+\frac{1}{2}}-\sum_{j=1}^{n}\left(a_{n-j}-a_{n-j+1}\right) v_{h}^{j-\frac{1}{2}}-\left(a_{n}-b_{n}\right) v_{h}^{\frac{1}{2}}-b_{n} v_{h}^{0}\right], v_{h}^{n+\frac{1}{2}}\right) \\
\leq & \frac{\Delta t^{-\alpha}}{2 \Gamma(2-\alpha)}\left(\sum_{j=1}^{n} a_{n-j}\left\|v_{h}^{j-\frac{1}{2}}\right\|^{2}-\sum_{j=1}^{n+1} a_{n-j+1}\left\|v_{h}^{j-\frac{1}{2}}\right\|^{2}+\widehat{b}_{h}\left\|v_{h}^{0}\right\|^{2}\right) .
\end{aligned}
$$

Combine (20) with (21) to obtain

$$
\begin{gathered}
\left\|v_{h}^{n+1}\right\|^{2}+\left\|\sigma_{h}^{n+1}\right\|^{2}+\Delta t\left\|v_{h}^{n+\frac{1}{2}}\right\|^{2}+\frac{\Delta t^{1-\alpha}}{\Gamma(2-\alpha)} \sum_{j=1}^{n+1} a_{n-j+1}\left\|v_{h}^{j-\frac{1}{2}}\right\|^{2} \\
\leq\left\|v_{h}^{n}\right\|^{2}+\left\|\sigma_{h}^{n}\right\|^{2}+\frac{\Delta t^{1-\alpha}}{\Gamma(2-\alpha)} \sum_{j=1}^{n} a_{n-j}\left\|v_{h}^{j-\frac{1}{2}}\right\|^{2}+\frac{\Delta t^{1-\alpha}}{\Gamma(2-\alpha)} \widehat{b}_{n}\left\|v_{h}^{0}\right\|^{2} \\
+C \Delta t\left(\left\|v_{h}^{n+1}\right\|^{2}+\left\|v_{h}^{n}\right\|^{2}+\left\|\sigma_{h}^{n+1}\right\|^{2}+\left\|\sigma_{h}^{n}\right\|^{2}+\left\|g\left(\mathbf{x}, t_{n+\frac{1}{2}}\right)\right\|^{2}\right) .
\end{gathered}
$$

We denote

$$
\Xi\left(v_{h}^{n+1}, \sigma_{h}^{n+1}\right)=\left\|v_{h}^{n+1}\right\|^{2}+\left\|\sigma_{h}^{n+1}\right\|^{2}+\frac{\Delta t^{1-\alpha}}{\Gamma(2-\alpha)} \sum_{j=1}^{n+1} a_{n-j+1}\left\|v_{h}^{j-\frac{1}{2}}\right\|^{2} .
$$


Remove the non-negative term to obtain

$$
\begin{aligned}
\Xi\left(v_{h}^{n+1}, \sigma_{h}^{n+1}\right) \leq & \left(\frac{1+\Delta t}{1-\Delta t}\right) \Xi\left(v_{h}^{n}, \sigma_{h}^{n}\right)+\frac{1}{1-\Delta t} \frac{\Delta t^{1-\alpha}}{\Gamma(2-\alpha)} \widehat{b}_{h}\left\|v_{h}^{0}\right\|^{2}+\frac{C \Delta t}{1-\Delta t}\left\|g\left(\mathbf{x}, t_{n+\frac{1}{2}}\right)\right\|^{2} \\
\leq & \left(\frac{1+\Delta t}{1-\Delta t}\right)^{2} \Xi\left(v_{h}^{n-1}, \sigma_{h}^{n-1}\right)+\frac{1}{1-\Delta t} \frac{\Delta t^{1-\alpha}}{\Gamma(2-\alpha)}\left\|v_{h}^{0}\right\|^{2} \sum_{j=0}^{1} \widehat{b}_{n-j}\left(\frac{1+\Delta t}{1-\Delta t}\right)^{j} \\
& +\frac{C \Delta t}{1-\Delta t} \sum_{j=0}^{1}\left(\frac{1+\Delta t}{1-\Delta t}\right)^{j}\left\|g\left(\mathbf{x}, t_{n-j+\frac{1}{2}}\right)\right\|^{2} \\
\leq & \cdots \cdots \\
\leq & \left(\frac{1+\Delta t}{1-\Delta t}\right)^{n} \Xi\left(v_{h}^{1}, \sigma_{h}^{1}\right)+\frac{1}{1-\Delta t} \frac{\Delta t^{1-\alpha}}{\Gamma(2-\alpha)}\left\|v_{h}^{0}\right\|^{2} \sum_{j=0}^{n-1} \widehat{b}_{n-j}\left(\frac{1+\Delta t}{1-\Delta t}\right)^{j} \\
& +\frac{C \Delta t}{1-\Delta t} \sum_{j=0}^{n-1}\left(\frac{1+\Delta t}{1-\Delta t}\right)^{j}\left\|g\left(\mathbf{x}, t_{n-j+\frac{1}{2}}\right)\right\|^{2} .
\end{aligned}
$$

Noting that $\left(\frac{1+\Delta t}{1-\Delta t}\right)>1, \Delta t=T / N \leq T / n$, we have

$$
\begin{aligned}
\left(\frac{1+\Delta t}{1-\Delta t}\right)^{n} & \leq\left(\frac{1+\Delta t}{1-\Delta t}\right)^{n+1} \leq \cdots \leq\left(1+\frac{2 \Delta t}{1-\Delta t}\right)^{\frac{T}{\Delta t}} \\
& \leq \lim _{\Delta t \rightarrow 0}\left(1+\frac{2 \Delta t}{1-\Delta t}\right)^{\frac{T(1-\Delta t)}{2 \Delta t} \frac{2}{1-\Delta t}}=e^{2}
\end{aligned}
$$

Further, noting that $\widehat{b}_{n-j}>0$ and using Lemma 5, we have

$$
\begin{aligned}
& \frac{1}{1-\Delta t} \frac{\Delta t^{1-\alpha}}{\Gamma(2-\alpha)}\left\|v_{h}^{0}\right\|^{2} \sum_{j=0}^{n-1} b_{n-j}\left(\frac{1+\Delta t}{1-\Delta t}\right)^{j}+\frac{C \Delta t}{1-\Delta t} \sum_{j=0}^{n-1}\left(\frac{1+\Delta t}{1-\Delta t}\right)^{j}\left\|g\left(\mathbf{x}, t_{n-j+\frac{1}{2}}\right)\right\|^{2} \\
\leq & \frac{e^{2}}{1-\Delta t} \frac{\Delta t^{1-\alpha}}{\Gamma(2-\alpha)}\left\|v_{h}^{0}\right\|^{2} \sum_{j=0}^{n-1} \widehat{b}_{n-j}+\frac{C \Delta t}{1-\Delta t} \sum_{j=0}^{n-1}\left\|g\left(\mathbf{x}, t_{n-j+\frac{1}{2}}\right)\right\|^{2} \\
\leq & C\left(\frac{T^{1-\alpha}}{\Gamma(2-\alpha)}\left\|v_{h}^{0}\right\|^{2}+\max _{1 \leq j \leq n}\left\{\left\|g\left(\mathbf{x}, t_{j+\frac{1}{2}}\right)\right\|^{2}\right\}\right) .
\end{aligned}
$$

Substitute (25) and (26) into (24) to arrive at

$$
\Xi\left(v_{h}^{n+1}, \sigma_{h}^{n+1}\right) \leq C\left(\Xi\left(v_{h}^{1}, \sigma_{h}^{1}\right)+\frac{T^{1-\alpha}}{\Gamma(2-\alpha)}\left\|v_{h}^{0}\right\|^{2}+\max _{1 \leq j \leq n}\left\{\left\|g\left(\mathbf{x}, t_{j+\frac{1}{2}}\right)\right\|^{2}\right\}\right) .
$$

Now, we estimate $\Xi\left(v_{h}^{1}, \sigma_{h}^{1}\right)$. Using (12) (c), taking $\chi_{h}=v_{h}^{\frac{1}{2}}$, and using CauchySchwarz inequality, we have

$$
\begin{aligned}
& -\left(\nabla \sigma_{h}^{\frac{1}{2}}, \nabla v_{h}^{\frac{1}{2}}\right) \\
= & -\left(P_{\Delta t} v_{h}^{\frac{1}{2}}, v_{h}^{\frac{1}{2}}\right)-\left(\frac{\Delta t^{-\alpha}}{\Gamma(2-\alpha)}\left[\left(\frac{1}{2}\right)^{-\alpha} v_{h}^{\frac{1}{2}}-\alpha\left(\frac{1}{2}\right)^{-\alpha} v_{h}^{0}\right], v_{h}^{\frac{1}{2}}\right)-\left\|v_{h}^{\frac{1}{2}}\right\|^{2}+\left(g\left(\mathbf{x}, t_{\frac{1}{2}}\right), v_{h}^{\frac{1}{2}}\right) \\
\leq & -\frac{\left\|v_{h}^{1}\right\|^{2}-\left\|v_{h}^{0}\right\|^{2}}{2 \Delta t}-\left(\frac{1}{2}\right)^{-\alpha} \frac{\Delta t^{-\alpha}}{\Gamma(2-\alpha)}\left\|v_{h}^{\frac{1}{2}}\right\|^{2} \\
& +\frac{\Delta t^{-\alpha}}{\Gamma(2-\alpha)} \alpha\left(\frac{1}{2}\right)^{-\alpha}\left(\left\|v_{h}^{0}\right\|^{2}+\left\|v_{h}^{\frac{1}{2}}\right\|^{2}\right)-\frac{1}{2}\left\|v_{h}^{\frac{1}{2}}\right\|^{2}+\frac{1}{2}\left\|g\left(\mathbf{x}, t_{\frac{1}{2}}\right)\right\|^{2} .
\end{aligned}
$$


For $n=0$, we sum for (18) and (28) to obtain

$$
\begin{aligned}
& \frac{\left\|v_{h}^{1}\right\|^{2}-\left\|v_{h}^{0}\right\|^{2}}{2 \Delta t}+\frac{\left\|\sigma_{h}^{1}\right\|^{2}-\left\|\sigma_{h}^{0}\right\|^{2}}{2 \Delta t}+\left(\frac{1}{2}+\frac{2^{\alpha} \Delta t^{-\alpha}}{\Gamma(1-\alpha)}\right)\left\|v_{h}^{\frac{1}{2}}\right\|^{2} \\
\leq & \frac{\alpha 2^{\alpha} \Delta t^{-\alpha}}{\Gamma(2-\alpha)}\left\|v_{h}^{0}\right\|^{2}+C\left(\left\|v_{h}^{1}\right\|^{2}+\left\|v_{h}^{0}\right\|^{2}+\left\|\sigma_{h}^{1}\right\|^{2}+\left\|\sigma_{h}^{0}\right\|^{2}+\left\|g\left(\mathbf{x}, t_{\frac{1}{2}}\right)\right\|^{2}\right) .
\end{aligned}
$$

Noting that $1-\alpha \leq 2^{\alpha},(0<\alpha<1)$ and (23), we have, for sufficiently small $\Delta t$,

$$
\begin{aligned}
\Xi\left(v_{h}^{1}, \sigma_{h}^{1}\right) & =\left\|v_{h}^{1}\right\|^{2}+\left\|\sigma_{h}^{1}\right\|^{2}+\frac{\Delta t^{1-\alpha}}{\Gamma(2-\alpha)} a_{0}\left\|v_{h}^{\frac{1}{2}}\right\|^{2} \\
& \leq\left\|v_{h}^{1}\right\|^{2}+\left\|\sigma_{h}^{1}\right\|^{2}+\left(1+\frac{2 \Delta t^{1-\alpha}}{\Gamma(1-\alpha)}\right)\left\|v_{h}^{\frac{1}{2}}\right\|^{2} \leq C\left(\left\|v_{h}^{0}\right\|^{2}+\left\|\sigma_{h}^{0}\right\|^{2}+\left\|g\left(\mathbf{x}, t_{\frac{1}{2}}\right)\right\|^{2}\right) .
\end{aligned}
$$

Substitute (30) into (27) to obtain

$$
\Xi\left(v_{h}^{n+1}, \sigma_{h}^{n+1}\right) \leq C\left(\left\|v_{h}^{0}\right\|^{2}+\left\|\sigma_{h}^{0}\right\|^{2}+\max _{0 \leq j \leq n}\left\{\left\|g\left(\mathbf{x}, t_{j+\frac{1}{2}}\right)\right\|^{2}\right\}\right), n \geq 0 .
$$

Combine (31) with (17) and use the Gronwall lemma to obtain

$$
\left\|u_{h}^{n+1}\right\|^{2} \leq C\left(\left\|u_{h}^{0}\right\|^{2}+\left\|v_{h}^{0}\right\|^{2}+\left\|\sigma_{h}^{0}\right\|^{2}+\max _{0 \leq j \leq n}\left\{\left\|g\left(\mathbf{x}, t_{j+\frac{1}{2}}\right)\right\|^{2}\right\}\right), n \geq 0 .
$$

Using (31) and (32), we obtain the conclusion.

\section{A Priori Error Estimate}

Now, we provide two projection operators [25] to derive a priori error estimates of our mixed finite element method.

Lemma 6. Define the $L^{2}$ projection $\mathcal{P}_{h}: L^{2}(\Omega) \rightarrow L_{h}$ as

$$
\left(u-\mathcal{P}_{h} u, \varphi_{h}\right)=0, \forall \varphi_{h} \in L_{h},
$$

with the estimate inequality

$$
\left\|u-\mathcal{P}_{h} u\right\|+\left\|u_{t}-\mathcal{P}_{h} u_{t}\right\| \leq C h^{m+1}\|u\|_{m+1}, \forall u \in L^{2}(\Omega) .
$$

Lemma 7. Define the elliptic projection $\mathcal{Q}_{h}: H_{0}^{1}(\Omega) \rightarrow V_{h}$ as

$$
\left(\nabla\left(v-\mathcal{Q}_{h} v\right), \nabla \phi_{h}\right)=0, \forall \phi_{h} \in V_{h},
$$

with the following inequality:

$$
\begin{aligned}
& \left\|v-\mathcal{Q}_{h} v\right\|+\left\|v_{t}-\mathcal{Q}_{h} v_{t}\right\|+h\left\|v-\mathcal{Q}_{h} v\right\|_{1} \leq C h^{k+1}\left(\|v\|_{k+1}+\left\|v_{t}\right\|_{k+1}\right), \\
& \forall v \in H_{0}^{1}(\Omega) \cap H^{k+1}(\Omega) .
\end{aligned}
$$

In what follows, we derive the proof of error estimates in $L^{2}$-norm in detail.

Theorem 2. For $\mathcal{P}_{h} u(0)=u_{h}^{0}, \mathcal{Q}_{h} v(0)=v_{h}^{0}$ and $\mathcal{Q}_{h} \sigma(0)=\sigma_{h}^{0}$, there exists a positive constant $C$ that is independent of space-time step length sizes $(h, \Delta t)$ and we have for $n \geq 0$

$$
\begin{aligned}
& \left\|u\left(t_{n+1}\right)-u_{h}^{n+1}\right\|+\left\|v\left(t_{n+1}\right)-v_{h}^{n+1}\right\|+\left\|\sigma\left(t_{n+1}\right)-\sigma_{h}^{n+1}\right\| \\
\leq & C\left[\left(1+\mu t_{n+\frac{1}{2}}^{1-\beta}\right) h^{k+1}+\Delta t^{3-\beta}+h^{m+1}\right],
\end{aligned}
$$


where, for the Caputo fractional derivative, we take $\mu$ as 0; for the Riemann-Liouville fractional derivative, we take $\mu$ as 1 .

Proof. For convenience, we write

$$
\begin{aligned}
& u\left(t_{n}\right)-u_{h}^{n}=\left(u\left(t_{n}\right)-\mathcal{P}_{h} u^{n}\right)+\left(\mathcal{P}_{h} u^{n}-u_{h}^{n}\right)=\mathcal{E}^{n}+\mathfrak{E}^{n}, \\
& v\left(t_{n}\right)-v_{h}^{n}=\left(v\left(t_{n}\right)-\mathcal{Q}_{h} v^{n}\right)+\left(\mathcal{Q}_{h} v^{n}-v_{h}^{n}\right)=\mathcal{F}^{n}+\mathfrak{F}^{n}, \\
& \sigma\left(t_{n}\right)-\sigma_{h}^{n}=\left(\sigma\left(t_{n}\right)-\mathcal{Q}_{h} \sigma^{n}\right)+\left(\mathcal{Q}_{h} \sigma^{n}-\sigma_{h}^{n}\right)=\mathcal{H}^{n}+\mathfrak{H}^{n} .
\end{aligned}
$$

Applying triangle inequality, we have

$$
\begin{aligned}
\left\|u\left(t_{n}\right)-u_{h}^{n}\right\| & \leq\left\|\mathcal{E}^{n}\right\|+\left\|\mathfrak{E}^{n}\right\|, \\
\left\|v\left(t_{n}\right)-v_{h}^{n}\right\| & \leq\left\|\mathcal{F}^{n}\right\|+\left\|\mathfrak{F}^{n}\right\|, \\
\left\|\sigma\left(t_{n}\right)-\sigma_{h}^{n}\right\| & \leq\left\|\mathcal{H}^{n}\right\|+\left\|\mathfrak{H}^{n}\right\| .
\end{aligned}
$$

Using Lemmas 6 and 7 , we arrive at the estimates of $\left\|\mathcal{E}^{n}\right\|,\left\|\mathcal{F}^{n}\right\|$, and $\left\|\mathcal{H}^{n}\right\|$. Consequently, in the discussion below, we only need to derive the estimates of $\left\|\mathfrak{E}^{n}\right\|,\left\|\mathfrak{F}^{n}\right\|$, and $\left\|\mathfrak{H}^{n}\right\|$. Using projections (33) and (35), we have error equations as follows:

$$
\left\{\begin{aligned}
(a) & \left(P_{\Delta t} \mathfrak{E}^{n+\frac{1}{2}}, \varphi_{h}\right)=-\left(P_{\Delta t} \mathcal{E}^{n+\frac{1}{2}}, \varphi_{h}\right)+\left(\mathcal{F}^{n+\frac{1}{2}}+\mathfrak{F}^{n+\frac{1}{2}}, \varphi_{h}\right)+\left(R_{1}^{n+\frac{1}{2}}, \varphi_{h}\right), \\
(b) & \left(P_{\Delta t} \mathfrak{H}^{n+\frac{1}{2}}, \psi_{h}\right)+\left(\nabla \mathfrak{F}^{n+\frac{1}{2}}, \nabla \psi_{h}\right) \\
= & -\left(\frac{f_{u}\left(u^{n+1}\right) v^{n+1}+f_{u}\left(u^{n}\right) v^{n}}{2}-\frac{f_{u}\left(u_{h}^{n+1}\right) v_{h}^{n+1}+f_{u}\left(u_{h}^{n}\right) v_{h}^{n}}{2}, \psi_{h}\right) \\
& -\left(P_{\Delta t} \mathcal{H}^{n+\frac{1}{2}}, \psi_{h}\right)+\left(R_{2}^{n+\frac{1}{2}}, \psi_{h}\right), \\
(c) & \left(P_{\Delta t} \mathfrak{F}^{n+\frac{1}{2}}, \chi_{h}\right)+\left(\frac { \Delta t ^ { - \alpha } } { \Gamma ( 2 - \alpha ) } \left[a_{0} \mathfrak{F}^{n+\frac{1}{2}}-\sum_{j=1}^{n}\left(a_{n-j}-a_{n-j+1}\right) \mathfrak{F}^{j-\frac{1}{2}}\right.\right. \\
& \left.\left.-\left(a_{n}-b_{n}\right) \mathfrak{F}^{\frac{1}{2}}-\widehat{b}_{n} \mathfrak{F}^{0}\right], \chi_{h}\right)+\left(\mathcal{F}^{n+\frac{1}{2}}+\mathfrak{F}^{n+\frac{1}{2}}, \psi_{h}\right)-\left(\nabla \mathfrak{H}^{n+\frac{1}{2}}, \nabla \chi_{h}\right) \\
= & -\left(P_{\Delta t} \mathcal{F}^{n+\frac{1}{2}}, \chi_{h}\right)-\left(\frac { \Delta t ^ { - \alpha } } { \Gamma ( 2 - \alpha ) } \left[a_{0} \mathcal{F}^{n+\frac{1}{2}}-\sum_{j=1}^{n}\left(a_{n-j}-a_{n-j+1}\right) \mathcal{F}^{j-\frac{1}{2}}\right.\right. \\
& \left.\left.-\left(a_{n}-b_{n}\right) \mathcal{F}^{\frac{1}{2}}-\widehat{b}_{n} \mathcal{F}^{0}\right], \chi_{h}\right)+\left(R_{3}^{n+\frac{1}{2}}, \chi_{h}\right) .
\end{aligned}\right.
$$

In (39), we set $\varphi_{h}=\mathfrak{E}^{n+\frac{1}{2}}, \chi_{h}=\mathfrak{F}^{n+\frac{1}{2}}$, and $\psi_{h}=\mathfrak{H}^{n+\frac{1}{2}}$, and add the resulting equations to obtain

$$
\begin{aligned}
& \left(P_{\Delta t} \mathfrak{E}^{n+\frac{1}{2}}, \mathfrak{E}^{n+\frac{1}{2}}\right)+\left(P_{\Delta t} \mathfrak{F}^{n+\frac{1}{2}}, \mathfrak{F}^{n+\frac{1}{2}}\right)+\left(P_{\Delta t} \mathfrak{H}^{n+\frac{1}{2}}, \mathfrak{H}^{n+\frac{1}{2}}\right) \\
& +\left(\frac{\Delta t^{-\alpha}}{\Gamma(2-\alpha)}\left[a_{0} \mathfrak{F}^{n+\frac{1}{2}}-\sum_{j=1}^{n}\left(a_{n-j}-a_{n-j+1}\right) \mathfrak{F}^{j-\frac{1}{2}}-\left(a_{n}-b_{n}\right) \mathfrak{F}^{\frac{1}{2}}-\widehat{b}_{n} \mathfrak{F}^{0}\right], \mathfrak{F}^{n+\frac{1}{2}}\right) \\
= & -\left(P_{\Delta t} \mathcal{E}^{n+\frac{1}{2}}, \mathfrak{E}^{n+\frac{1}{2}}\right)-\left(P_{\Delta t} \mathcal{F}^{n+\frac{1}{2}}, \mathfrak{F}^{n+\frac{1}{2}}\right)-\left(P_{\Delta t} \mathcal{H}^{n+\frac{1}{2}}, \mathfrak{H}^{n+\frac{1}{2}}\right) \\
& +\left(\mathcal{F}^{n+\frac{1}{2}}+\mathfrak{F}^{n+\frac{1}{2}}, \mathfrak{E}^{n+\frac{1}{2}}\right)+\left(\mathcal{F}^{n+\frac{1}{2}}+\mathfrak{F}^{n+\frac{1}{2}}, \mathfrak{F}^{n+\frac{1}{2}}\right) \\
& -\left(\frac{f_{u}\left(u^{n+1}\right) v^{n+1}+f_{u}\left(u^{n}\right) v^{n}}{2}-\frac{f_{u}\left(u_{h}^{n+1}\right) v_{h}^{n+1}+f_{u}\left(u_{h}^{n}\right) v_{h}^{n}}{2}, \mathfrak{H}^{n+\frac{1}{2}}\right) \\
& -\left(\frac{\Delta t^{-\alpha}}{\Gamma(2-\alpha)}\left[a_{0} \mathcal{F}^{n+\frac{1}{2}}-\sum_{j=1}^{n}\left(a_{n-j}-a_{n-j+1}\right) \mathcal{F}^{j-\frac{1}{2}}-\left(a_{n}-b_{n}\right) \mathcal{F}^{\frac{1}{2}}-\widehat{b}_{n} \mathcal{F}^{0}\right], \mathfrak{F}^{n+\frac{1}{2}}\right) \\
& +\left(R_{1}^{n+\frac{1}{2}}, \mathfrak{E}^{n+\frac{1}{2}}\right)+\left(R_{2}^{n+\frac{1}{2}}, \mathfrak{H}^{n+\frac{1}{2}}\right)+\left(R_{3}^{n+\frac{1}{2}}, \mathfrak{F}^{n+\frac{1}{2}}\right) .
\end{aligned}
$$


Now, we need to estimate all terms on the right-hand side of (40). Using CauchySchwarz inequality, we have

$$
\begin{aligned}
& -\left(P_{\Delta t} \mathcal{E}^{n+\frac{1}{2}}, \mathfrak{E}^{n+\frac{1}{2}}\right)-\left(P_{\Delta t} \mathcal{F}^{n+\frac{1}{2}}, \mathfrak{F}^{n+\frac{1}{2}}\right)-\left(P_{\Delta t} \mathcal{H}^{n+\frac{1}{2}}, \mathfrak{H}^{n+\frac{1}{2}}\right) \\
& +\left(\mathcal{F}^{n+\frac{1}{2}}+\mathfrak{F}^{n+\frac{1}{2}}, \mathfrak{E}^{n+\frac{1}{2}}\right)+\left(\mathcal{F}^{n+\frac{1}{2}}+\mathfrak{F}^{n+\frac{1}{2}}, \mathfrak{F}^{n+\frac{1}{2}}\right) \\
\leq & C\left(\left\|P_{\Delta t} \mathcal{E}^{n+\frac{1}{2}}\right\|^{2}+\left\|P_{\Delta t} \mathcal{F}^{n+\frac{1}{2}}\right\|^{2}+\left\|P_{\Delta t} \mathcal{H}^{n+\frac{1}{2}}\right\|^{2}+\left\|\mathcal{F}^{n+\frac{1}{2}}\right\|^{2}\right) \\
& +C\left(\left\|\mathfrak{E}^{n+\frac{1}{2}}\right\|^{2}+\left\|\mathfrak{F}^{n+\frac{1}{2}}\right\|^{2}+\left\|\mathfrak{H}^{n+\frac{1}{2}}\right\|^{2}\right) .
\end{aligned}
$$

Applying the mean value theorem and Cauchy-Schwarz inequality, we have

$$
\begin{aligned}
& -\left(\frac{f_{u}\left(u^{n+1}\right) v^{n+1}+f_{u}\left(u^{n}\right) v^{n}}{2}-\frac{f_{u}\left(u_{h}^{n+1}\right) v_{h}^{n+1}+f_{u}\left(u_{h}^{n}\right) v_{h}^{n}}{2}, \mathfrak{H}^{n+\frac{1}{2}}\right) \\
= & -\frac{1}{2}\left(f_{u}\left(u^{n+1}\right)\left(v^{n+1}-v_{h}^{n+1}\right)+\left(f_{u}\left(u^{n+1}\right)-f_{u}\left(u_{h}^{n+1}\right)\right) v_{h}^{n+1}\right. \\
& \left.+f_{u}\left(u^{n}\right)\left(v^{n}-v_{h}^{n}\right)+\left(f_{u}\left(u^{n}\right)-f_{u}\left(u_{h}^{n}\right)\right) v_{h}^{n}, \mathfrak{H}^{n+\frac{1}{2}}\right) \\
\leq & \frac{1}{2}\left(\left\|f_{u}\left(u^{n+1}\right)\right\|_{\infty}\left\|v^{n+1}-v_{h}^{n+1}\right\|+\left\|f_{u u}\left(\bar{\theta}^{n+1}\right)\right\|_{\infty}\left\|u^{n+1}-u_{h}^{n+1}\right\|\left\|v_{h}^{n+1}\right\|_{\infty}\right. \\
& \left.+\left\|f_{u}\left(u^{n}\right)\right\|\left\|_{\infty}\right\| v^{n}-v_{h}^{n}\|+\| f_{u u}\left(\bar{\theta}^{n}\right)\|\infty\| u^{n}-u_{h}^{n}\|\| v_{h}^{n} \|_{\infty}\right)\left\|\mathfrak{H}^{n+\frac{1}{2}}\right\| . \\
\leq & C\left(\left\|\mathcal{E}^{n+1}\right\|^{2}+\left\|\mathcal{F}^{n+1}\right\|^{2}+\left\|\mathcal{E}^{n}\right\|^{2}+\left\|\mathcal{F}^{n}\right\|^{2}+\left\|\mathfrak{E}^{n+1}\right\|^{2}\right. \\
& \left.+\left\|\mathfrak{F}^{n+1}\right\|^{2}+\left\|\mathfrak{H}^{n+1}\right\|^{2}+\left\|\mathfrak{E}^{n}\right\|^{2}+\left\|\mathfrak{F}^{n}\right\|^{2}+\left\|\mathfrak{H}^{n}\right\|^{2}\right),
\end{aligned}
$$

where we use the boundedness of $\left\|f_{u}\left(u^{n}\right)\right\|_{\infty}$ and the following bounded inequality:

$$
\left\|f_{u u}\left(\bar{\theta}^{n}\right)\right\|_{\infty}+\left\|v_{h}^{n}\right\|_{\infty} \leq C,
$$

where one can apply inverse inequality [25], and use a similar method as the one in [7,26].

Making use of (9), (3), Cauchy-Schwarz inequality, as well as Young inequality, we have

$$
\begin{aligned}
& -\left(\frac{\Delta t^{-\alpha}}{\Gamma(2-\alpha)}\left[a_{0} \mathcal{F}^{n+\frac{1}{2}}-\sum_{j=1}^{n}\left(a_{n-j}-a_{n-j+1}\right) \mathcal{F}^{j-\frac{1}{2}}-\left(a_{n}-b_{n}\right) \mathcal{F}^{\frac{1}{2}}-\widehat{b}_{n} \mathcal{F}^{0}\right], \mathfrak{F}^{n+\frac{1}{2}}\right) \\
& +\left(R_{1}^{n+\frac{1}{2}}, \mathfrak{E}^{n+\frac{1}{2}}\right)+\left(R_{2}^{n+\frac{1}{2}}, \mathfrak{H}^{n+\frac{1}{2}}\right)+\left(R_{3}^{n+\frac{1}{2}}, \mathfrak{F}^{n+\frac{1}{2}}\right) \\
= & -\left(\frac{1}{\Gamma(1-\alpha)} \int_{0}^{t}{ }^{t+\frac{1}{2}} \frac{\frac{\partial \mathcal{F}}{\partial s} d s}{\left(t_{n+\frac{1}{2}}-s\right)^{\alpha}}+\frac{\mu \mathcal{F}^{0}}{\Gamma(1-\alpha)} t_{n+\frac{1}{2}}^{-\alpha}+O\left(\Delta t^{2-\alpha}\right), \mathfrak{F}^{n+\frac{1}{2}}\right) \\
& +\left(R_{1}^{n+\frac{1}{2}}, \mathfrak{E}^{n+\frac{1}{2}}\right)+\left(R_{2}^{n+\frac{1}{2}}, \mathfrak{H}^{n+\frac{1}{2}}\right)+\left(R_{3}^{n+\frac{1}{2}}, \mathfrak{F}^{n+\frac{1}{2}}\right) \\
\leq & C\left[\left(1+\mu t_{n+\frac{1}{2}}^{-\alpha} h^{2 k+2}+\Delta t^{4-2 \alpha}+\left\|\mathfrak{E}^{n+\frac{1}{2}}\right\|^{2}+\left\|\mathfrak{F}^{n+\frac{1}{2}}\right\|^{2}+\left\|\mathfrak{H}^{n+\frac{1}{2}}\right\|^{2}\right] .\right.
\end{aligned}
$$


Making a combination for (41)-(44) and using (18), we have

$$
\begin{aligned}
& \frac{\left(\left\|\mathfrak{E}^{n+1}\right\|^{2}+\left\|\mathfrak{F}^{n+1}\right\|^{2}+\left\|\mathfrak{H}^{n+1}\right\|^{2}\right)-\left(\left\|\mathfrak{E}^{n}\right\|^{2}+\left\|\mathfrak{F}^{n}\right\|^{2}+\left\|\mathfrak{H}^{n}\right\|^{2}\right)}{2 \Delta t} \\
& +\frac{\Delta t^{-\alpha}}{2 \Gamma(2-\alpha)} \sum_{j=1}^{n+1} a_{n-j+1}\left\|\mathfrak{F}^{j-\frac{1}{2}}\right\|^{2} \\
= & \frac{\Delta t^{-\alpha}}{2 \Gamma(2-\alpha)} \sum_{j=1}^{n} a_{n-j}\left\|\mathfrak{F}^{j-\frac{1}{2}}\right\|^{2}+\frac{\Delta t^{-\alpha}}{2 \Gamma(2-\alpha)} \widehat{b}_{n}\left\|\mathfrak{F}^{0}\right\|^{2}+C\left[\left(1+\mu t_{n+\frac{1}{2}}^{-\alpha}\right) h^{2 k+2}+\Delta t^{4-2 \alpha}\right. \\
& +\left\|P_{\Delta t} \mathcal{E}^{n+\frac{1}{2}}\right\|^{2}+\left\|P_{\Delta t} \mathcal{F}^{n+\frac{1}{2}}\right\|^{2}+\left\|P_{\Delta t} \mathcal{H}^{n+\frac{1}{2}}\right\|^{2}+\left\|\mathcal{E}^{n+1}\right\|^{2}+\left\|\mathcal{F}^{n+1}\right\|^{2} \\
& \left.+\left\|\mathcal{E}^{n}\right\|^{2}+\left\|\mathcal{F}^{n}\right\|^{2}+\left\|\mathfrak{E}^{n+1}\right\|^{2}+\left\|\mathfrak{F}^{n+1}\right\|^{2}+\left\|\mathfrak{H}^{n+1}\right\|^{2}+\left\|\mathfrak{E}^{n}\right\|^{2}+\left\|\mathfrak{F}^{n}\right\|^{2}+\left\|\mathfrak{H}^{n}\right\|^{2}\right] .
\end{aligned}
$$

With given conditions $\mathfrak{E}^{0}=0, \mathfrak{F}^{0}=0, \mathfrak{H}^{0}=0$, we use (23) to arrive at

$$
\begin{aligned}
& \Xi\left(\mathfrak{F}^{n+1}, \mathfrak{H}^{n+1}\right)+\left\|\mathfrak{E}^{n+1}\right\|^{2} \\
\leq & \Xi\left(\mathfrak{F}^{n}, \mathfrak{H}^{n}\right)+\left\|\mathfrak{E}^{n}\right\|^{2}+C \Delta t\left[\left(1+\mu t_{n+\frac{1}{2}}^{-\alpha}\right) h^{2 k+2}+\Delta t^{4-2 \alpha}\right. \\
& \left.+h^{2 m+2}+\left\|\mathfrak{E}^{n+1}\right\|^{2}+\left\|\mathfrak{F}^{n+1}\right\|^{2}+\left\|\mathfrak{H}^{n+1}\right\|^{2}+\left\|\mathfrak{E}^{n}\right\|^{2}+\left\|\mathfrak{F}^{n}\right\|^{2}+\left\|\mathfrak{H}^{n}\right\|^{2}\right] .
\end{aligned}
$$

Sum for (46) with respect to $n$ to arrive at

$$
\begin{aligned}
& \Xi\left(\mathfrak{F}^{n+1}, \mathfrak{H}^{n+1}\right)+\left\|\mathfrak{E}^{n+1}\right\|^{2} \\
\leq & \Xi\left(\mathfrak{F}^{1}, \mathfrak{H}^{1}\right)+\left\|\mathfrak{E}^{1}\right\|^{2}+C \Delta t \sum_{j=1}^{n}\left[\left(1+\mu t_{j+\frac{1}{2}}^{-\alpha}\right) h^{2 k+2}+\Delta t^{4-2 \alpha}+h^{2 m+2}\right] \\
& +C \Delta t \sum_{j=1}^{n+1}\left[\left\|\mathfrak{E}^{j}\right\|^{2}+\left\|\mathfrak{F}^{j}\right\|^{2}+\left\|\mathfrak{H}^{j}\right\|^{2}\right] .
\end{aligned}
$$

For $n=0$, we use a similar derivation to the one of $n \geq 1$ and apply triangle inequality to arrive at

$$
\Xi\left(\mathfrak{F}^{1}, \mathfrak{H}^{1}\right)+\left\|\mathfrak{E}^{1}\right\|^{2} \leq C\left[\left(1+\mu t_{\frac{1}{2}}^{-\alpha}\right) h^{2 k+2}+\Delta t^{4-2 \alpha}+h^{2 m+2}\right]
$$

Substitute (48) into (47) and use the Gronwall lemma to obtain

$$
\Xi\left(\mathfrak{F}^{n+1}, \mathfrak{H}^{n+1}\right)+\left\|\mathfrak{E}^{n+1}\right\|^{2} \leq C\left[\left(1+\mu t_{n+\frac{1}{2}}^{-\alpha}\right) h^{2 k+2}+\Delta t^{4-2 \alpha}+h^{2 m+2}\right], \forall n \geq 0 .
$$

Combining (49), (34), and (36) with (38) and noting that $\alpha=\beta-1$, we complete the proof of the theorem.

Remark 2. Compared with the classical mixed element method for fourth-order partial differential equations, our method can approximate simultaneously three variables with optimal error estimates in $L^{2}$-norm. More importantly, we can obtain directly optimal error estimates in $L^{2}$-norm for auxiliary variables in solving fourth-order PDEs, which are difficult to achieve by using classical mixed element methods [6-8].

\section{Numerical Tests}

Here, we will verify the theoretical results by numerical computing. In (1), we take space domain $\bar{\Omega}=[0,1]^{2}$, time interval $\bar{J}=[0,1]$, nonlinear term $f(u)=u^{3}-u$, initial conditions with $u(x, y, 0)=0, u_{1}(x, y)=0$, and exact solution $u=t^{3} \sin (2 \pi x) \sin (2 \pi y)$; we can obtain the source term $g(x, y, t)$ and two auxiliary variables $v=3 t^{2} \sin (2 \pi x) \sin (2 \pi y)$ and $\sigma=-t^{3} \sin (2 \pi x) \sin (2 \pi y)\left(8 \pi^{2}+t^{6} \sin (2 \pi x)^{2} \sin (2 \pi y)^{2}-1\right)$. In the following numer- 
ical calculations, the order of convergence in space is calculated by the following formula with a sufficiently small time step size $\Delta t$

$$
\text { Order }=\log _{\frac{h_{1}}{h_{2}}} \frac{\left\|\phi-\phi_{h_{1}}\right\|}{\left\|\phi-\phi_{h_{2}}\right\|}
$$

where $h_{k}(k=1,2)$ represents different space mesh step lengths.

For implementing the new mixed element algorithm, we approximate the spatial direction by the finite element method with the basis function $P(x, y)=a+b x+c y+d x y$ and discretize the time direction by using the modified $L 1$ Crank-Nicolson scheme. In Table 1 , by taking the fixed time mesh parameter $\Delta t=1 / 200$, changed spatial step length sizes $h=\sqrt{2} / 9, \sqrt{2} / 16$ and $\sqrt{2} / 25$, and different parameters $\beta=1.1,1.5,1.9$, we show the $L^{2}$-norm error estimates and second-order convergence data in space. In Tables 2 and 3, we compute the convergence results $v$ and $\sigma$, respectively. From Tables 1-3, one can see that the numerical method is effective for solving nonlinear fourth-order fractional diffusion-wave equation models with a smooth solution.

Table 1. The convergence results for $u$ with $\Delta t=1 / 200$.

\begin{tabular}{ccccc}
\hline$\beta$ & $h$ & $\left\|u-u_{h}\right\|$ & Order & CPU-Time (s) \\
\hline 1.1 & $\sqrt{2} / 9$ & $4.1181 \times 10^{-2}$ & & 1.13 \\
& $\sqrt{2} / 16$ & $1.3341 \times 10^{-2}$ & 1.9590 & 4.04 \\
& $\sqrt{2} / 25$ & $5.5001 \times 10^{-3}$ & 1.9855 & 19.03 \\
1.5 & $\sqrt{2} / 9$ & $4.1175 \times 10^{-2}$ & & 1.10 \\
& $\sqrt{2} / 16$ & $1.3339 \times 10^{-2}$ & 1.9590 & 4.02 \\
& $\sqrt{2} / 25$ & $5.4991 \times 10^{-3}$ & 1.9855 & 19.40 \\
1.9 & $\sqrt{2} / 9$ & $4.1169 \times 10^{-2}$ & & 4.14 \\
& $\sqrt{2} / 16$ & $1.3336 \times 10^{-2}$ & 1.9591 & 19.33 \\
\hline
\end{tabular}

Table 2. The convergence results for $v$ with $\Delta t=1 / 200$.

\begin{tabular}{ccccc}
\hline $\boldsymbol{\beta}$ & $\boldsymbol{h}$ & $\left\|v-v_{h}\right\|$ & Order & CPU-Time (s) \\
\hline 1.1 & $\sqrt{2} / 9$ & $1.2293 \times 10^{-1}$ & & 1.13 \\
& $\sqrt{2} / 16$ & $3.9815 \times 10^{-2}$ & 1.9594 & 4.04 \\
& $\sqrt{2} / 25$ & $1.6417 \times 10^{-2}$ & 1.9851 & 19.03 \\
1.5 & $\sqrt{2} / 9$ & $1.2292 \times 10^{-1}$ & & 1.10 \\
& $\sqrt{2} / 16$ & $3.9816 \times 10^{-2}$ & 1.9593 & 4.02 \\
& $\sqrt{2} / 25$ & $1.6417 \times 10^{-2}$ & 1.9852 & 19.40 \\
1.9 & $\sqrt{2} / 9$ & $1.2293 \times 10^{-1}$ & & 4.14 \\
& $\sqrt{2} / 16$ & $3.9819 \times 10^{-2}$ & 1.9592 & 19.33 \\
\hline
\end{tabular}

Table 3. The convergence results for $\sigma$ with $\Delta t=1 / 200$.

\begin{tabular}{ccccc}
\hline $\boldsymbol{\beta}$ & $h$ & $\left\|\sigma-\sigma_{h}\right\|$ & Order & CPU-Time (s) \\
\hline 1.1 & $\sqrt{2} / 9$ & $1.8858 \times 10^{+0}$ & & 1.13 \\
& $\sqrt{2} / 16$ & $5.9584 \times 10^{-1}$ & 2.0024 & 4.04 \\
& $\sqrt{2} / 25$ & $2.4398 \times 10^{-1}$ & 2.0007 & 19.03 \\
1.5 & $\sqrt{2} / 9$ & $1.8853 \times 10^{+0}$ & & 1.10 \\
& $\sqrt{2} / 16$ & $5.9568 \times 10^{-1}$ & 2.0025 & 4.02 \\
& $\sqrt{2} / 25$ & $2.4391 \times 10^{-1}$ & 2.0007 & 19.40 \\
1.9 & $\sqrt{2} / 9$ & $1.8849 \times 10^{+0}$ & & 1.14 \\
& $\sqrt{2} / 16$ & $5.9550 \times 10^{-1}$ & 2.0026 & 4.07 \\
& $\sqrt{2} / 25$ & $2.4381 \times 10^{-1}$ & 2.0010 & 19.33 \\
\hline
\end{tabular}




\title{
5. Concluding Remarks
}

From the calculated results in Tables 1-3, one can see that our method for solving fourth-order fractional diffusion-wave equations in this article can obtain optimal error estimates in $L^{2}$-norm for three variables, which is in agreement with the derived theoretical results. These results for auxiliary variables are difficult to achieve directly by using classical mixed element methods [6-8].

In the future, we will improve our mixed element method by combining other techniques $[7,27,28]$ with high-order time approximate schemes and develop their optimal numerical theories.

Author Contributions: Conceptualization, J.W.; methodology, J.W., Y.L. and H.L.; software, B.Y.; validation, Y.L., H.L. and Z.F.; formal analysis, J.W.; writing — original draft preparation, J.W.; writingreview and editing, Y.L., H.L. and Z.F.; funding acquisition, J.W., Y.L., H.L. and Z.F. All authors have read and agreed to the published version of the manuscript.

Funding: This work is supported by the National Natural Science Foundation of China (12061053, 12161063), Natural Science Foundation of Inner Mongolia (2020MS01003, 2021MS01018), Young Innovative Talents Project of Grassland Talents Project, Scientific Research Projection of Higher Schools of Inner Mongolia (NJZY21266), and Program for Innovative Research Team in Universities of Inner Mongolia Autonomous Region (NMGIRT2207).

Institutional Review Board Statement: Not applicable.

Informed Consent Statement: Not applicable.

Data Availability Statement: All the data are computed by using our mixed element method.

Conflicts of Interest: The authors declare no conflict of interest.The funders had no role in the design of the study; in the collection, analyses, or interpretation of data; in the writing of the manuscript, or in the decision to publish the results.

\author{
Abbreviations \\ The following abbreviations are used in this manuscript: \\ MDPI Multidisciplinary Digital Publishing Institute \\ DOAJ Directory of Open Access Journals \\ TLA Three-letter acronym \\ LD Linear dichroism
}

\section{References}

1. Nikan, O.; Tenreiro Machado, J.A.; Golbabai, A. Numerical solution of time-fractional fourth-order reaction-diffusion model arising in composite environments. Appl. Math. Model. 2021, 89, 819-836. [CrossRef]

2. Hu, X.L.; Zhang, L.M. On finite difference methods for fourth-order fractional diffusion-wave and subdiffusion systems. Appl. Math. Comput. 2012, 218, 5019-5034. [CrossRef]

3. Liu, Y.; Fang, Z.C.; Li, H.; He, S. A mixed finite element method for a time-fractional fourth-order partial differential equation. Appl. Math. Comput. 2014, 243, 703-717. [CrossRef]

4. Agrawal, O.P. A general solution for a fourth-order fractional diffusion-wave equation defined in a bounded domain. Comput. Struct. 2001, 79, 1497-1501. [CrossRef]

5. Jafari, H.; Dehghan, M.; Sayevand, K. Solving a fourth-order fractional diffusion-wave equation in a bounded domain by decomposition method. Numer. Meth. Part. Differ. Equ. 2008, 24, 1115-1126. [CrossRef]

6. Liu, N.; Liu, Y.; Li, H.; Wang, J.F. Time second-order finite difference/finite element algorithm for nonlinear time-fractional diffusion problem with fourth-order derivative term. Comput. Math. Appl. 2018, 75, 3521-3536.

7. Liu, Y.; Du, Y.W.; Li, H.; Li, J.C.; He, S. A two-grid mixed finite element method for a nonlinear fourth-order reaction-diffusion problem with time-fractional derivative. Comput. Math. Appl. 2015, 70, 2474-2492. [CrossRef]

8. Liu, Y.; Du, Y.W.; Li, H.; He, S.; Gao, W. Finite difference/finite element method for a nonlinear time-fractional fourth-order reaction-diffusion problem. Comput. Math. Appl. 2015, 70, 573-591. [CrossRef]

9. Ji, C.C.; Sun, Z.Z.; Hao, Z.P. Numerical algorithms with high spatial accuracy for the fourth-order fractional sub-diffusion equations with the first Dirichlet boundary conditions. J. Sci. Comput. 2016, 66, 1148-1174. [CrossRef] 
10. Ran, M.H.; Zhang, C.J. New compact difference scheme for solving the fourth-order time fractional sub-diffusion equation of the distributed order. Appl. Numer. Math. 2018, 129, 58-70. [CrossRef]

11. Nandal, S.; Pandey, D.N. Numerical solution of non-linear fourth order fractional sub-diffusion wave equation with time delay. Appl. Math. Comput. 2020, 369, 124900. [CrossRef]

12. Sun, H.; Sun, Z.Z.; Du, R. A linearized second-order difference scheme for the nonlinear time-fractional fourth-order reactiondiffusion equation. Numer. Math. Theory Meth. Appl. 2019, 12, 1168-1190.

13. Huang, J.F.; Qiao, Z.; Zhang, J.N.; Arshad, S.; Tang, Y.F. Two linearized schemes for time fractional nonlinear wave equations with fourth-order derivative. J. Appl. Math. Comput. 2021, 66, 561-579. [CrossRef]

14. Abbaszadeh, M.; Dehghan, M. Direct meshless local Petrov-Galerkin (DMLPG) method for time-fractional fourth-order reaction-diffusion problem on complex domains. Comput. Math. Appl. 2020, 79, 876-888. [CrossRef]

15. Yang, X.H.; Zhang, H.X.; Xu, D. Orthogonal spline collocation method for the fourth-order diffusion system. Comput. Math. Appl. 2018, 75, 3172-3185. [CrossRef]

16. Zhang, H.X.; Yang, X.H.; Xu, D. A high-order numerical method for solving the 2D fourth-order reaction-diffusion equation. Numer. Algor. 2019, 80, 849-877. [CrossRef]

17. Tariq, H.; Akram, G. Quintic spline technique for time fractional fourth-order partial differential equation. Numer. Meth. Part Differ. Equ. 2017, 33, 445-466. [CrossRef]

18. Guo, L.; Wang, Z.B.; Vong, S.K. Fully discrete local discontinuous Galerkin methods for some time-fractional fourth-order problems. Int. J. Comput. Math. 2016, 93, 1665-1682. [CrossRef]

19. Du, Y.W.; Liu, Y.; Li, H.; Fang, Z.C.; He, S. Local discontinuous Galerkin method for a nonlinear time-fractional fourth-order partial differential equation. J. Comput. Phys. 2017, 344, 108-126. [CrossRef]

20. Li, X.H.; Wong, P.J.Y. An efficient numerical treatment of fourth-order fractional diffusion-wave problems. Numer. Meth. Part. Differ. Equ. 2018, 34, 1324-1347. [CrossRef]

21. Grasselli, M.; Pierre, M. A splitting method for the Cahn-Hilliard equation with inertial term. Math. Model. Meth. Appl. Sci. 2010, 20, 1363-1390. [CrossRef]

22. Zeng, F.H.; Li, C.P. A new Crank-Nicolson finite element method for the time-fractional subdiffusion equation. Appl. Numer. Math. 2017, 121, 82-95. [CrossRef]

23. Sun, Z.Z.; Wu, X.N. A fully discrete difference scheme for a diffusion-wave system. Appl. Numer. Math. 2006, 56, 193-209. [CrossRef]

24. Lin, Y.M.; Xu, C.J. Finite difference/spectral approximations for the time-fractional diffusion equation. J. Comput. Phys. 2007, 225, 1533-1552. [CrossRef]

25. Ciarlet, P.G. The Finite Element Method for Elliptic Problems; Elsevier: Amsterdam, The Netherlands, 1978.

26. Li, D.F.; Zhang, J.W.; Zhang, Z.M. Unconditionally optimal error estimates of a linearized galerkin method for nonlinear time fractional reaction-subdiffusion equations. J. Sci. Comput. 2018, 76, 848-866. [CrossRef]

27. Bu, W.P.; Liu, X.T.; Tang, Y.F.; Yang, J.Y. Finite element multigrid method for multi-term time fractional advection diffusion equations. Int. J. Model. Simulat. Sci. Comput. 2015, 6, 1540001. [CrossRef]

28. Yue, X.Q.; Shu, S.; Xu, X.; Bu, W.; Pan, K. Parallel-in-time multigrid for space-time finite element approximations of twodimensional space-fractional diffusion equations. Comput. Math. Appl. 2019, 78, 3471-3484. [CrossRef] 